\section{§8. Design Study of an Indirect Cooling Superconducting Magnet for a Fusion Device}

Mito, T.,

Hemmi, T. (JAEA)

The design study of superconducting magnets adapting the indirect cooling method is reported. The superconducting magnet system for the spherical tokamak (ST), which is proposed to study the steady state plasma experiment with Q_equiv $\sim 1$, requires high performances with a high current density compared to the ordinal magnet design because of its tight spatial restriction. The superconducting magnet system for the fusion device has been used in the condition of high magnetic field, high electromagnetic force, and high heat load. The pool boiling of liquid helium cooling outside of the conductor or the forced flow of supercritical helium cooling inside of the conductor such as cable-in-conduit conductors were used so far for the cooling method of the superconducting magnet for a fusion application. The pool cooling magnet has the disadvantages of low mechanical rigidities and low withstand voltages of the coil windings. On the other hand, the forced flow cooling magnet with cable-in-conduit conductors has the disadvantages of the restriction of the coil design because of the path of the electric current must be the same as that of the cooling channel for refrigerant.

The path of the electric current and cooling channel for refrigerant can be independently designed by adopting the indirect cooling method that inserts the independent cooling panel in the coil windings and cools the conductor from the outside. Therefore the optimization of the coil windings structure can be attempted. The dimension and the configuration of the ST system composed of eight D-shape TF coils are shown in Fig. 1. The cross section of the TF coil has a sector shape as shown in Fig. 2 The coil windings are composed of four double pancake coils, and covered with the stainless steel coil case to support a large electromagnetic force. The superconducting conductor for indirect cooling is composed of a transposed bundle of $\mathrm{Nb}_{3} \mathrm{Al}$ wires jacketed with aluminum alloy as shown in Fig. 3. The conductors in the coil windings are indirectly cooled by the cooling panels which are inserted in the coil windings. In the cooling panel, the cooling channels are imbetted for forced flow cooling with supercritical helium.

The temperature rise in the coil windings is calculated by ANSYSTM at the condition with the steady heat load of $100 \mathrm{~W} / \mathrm{m}^{3}$ assuming the temperature of the cooling panel is fixed to $5 \mathrm{~K}$. The maximum temperature in the coil windings is only $5.15 \mathrm{~K}$, which indicates a high thermal performance of the indirectly cooled coil. The mechanical characteristics for the large electromagnetic force and the safety margin against the coil quench are also examined with a parameter of the coil current density. As a result, the high current density of $60 \mathrm{~A} / \mathrm{mm}^{2}$ is possible by using the indirect cooling method. It was shown that the superconducting magnet design of the high current density becomes possible by the indirect cooling method compared with those of the conventional cooling scheme.

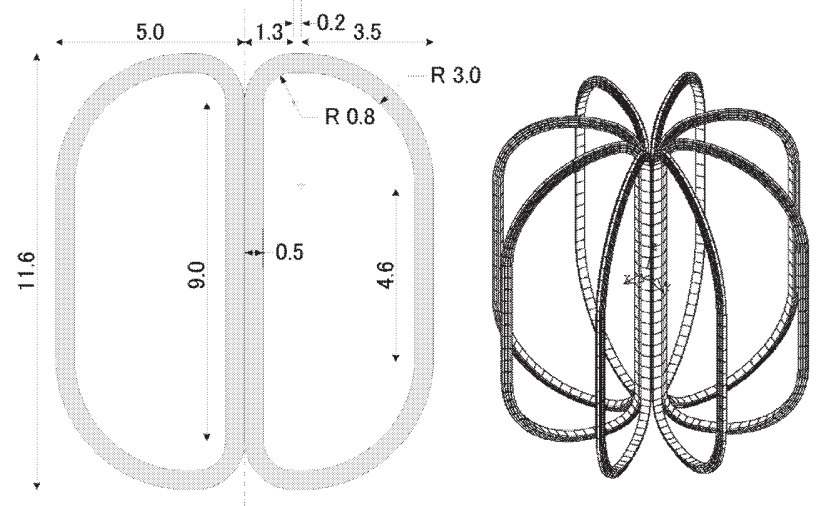

Fig. 1. TF coil dimension and configuration for the ST device $0.5 \mathrm{~m}$

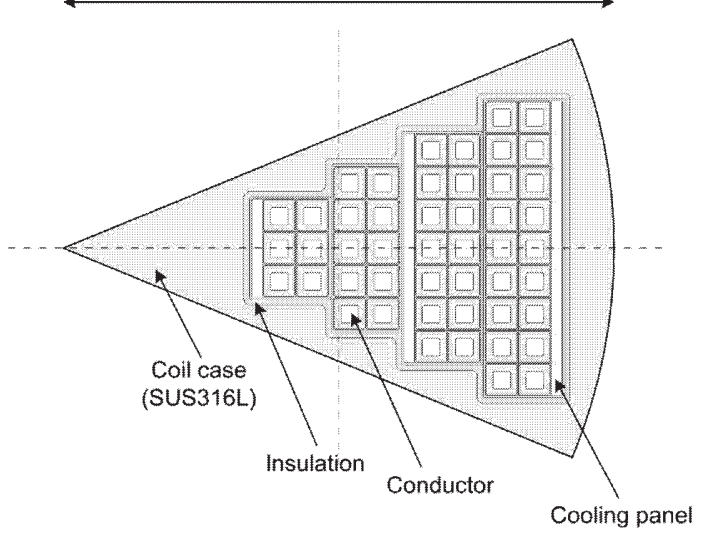

Fig. 2. Cross section of the TF coil by indirect cooling.

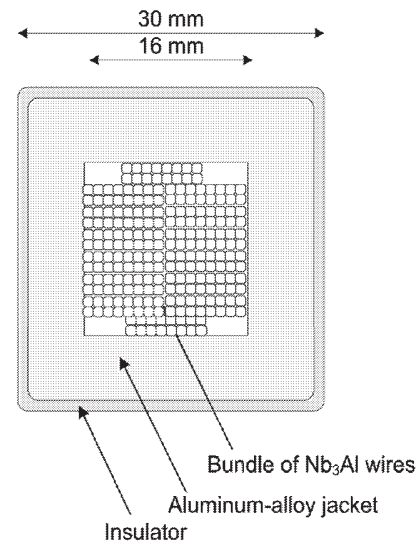

Fig. 3. Cross section of the indirectly cooled superconducting conductor for the TF coil.

1) Mito, T., Hemmi, T., " Design study of an indirect cooling superconducting magnet for a fusion device", to be published on IEEJ Trans., Fundamentals and Materials (in Japanese). 